\title{
P System with Chain Structure and Simulation in Arithmetic Operation
}

\author{
Jing Luan ${ }^{1, \mathrm{a}}$, Zhong Yao ${ }^{1, \mathrm{~b}}$ \\ ${ }^{1}$ School of Economics and Management, Beihang University, Beijing \\ 100191, China \\ aluanjing1408@buaa.edu.cn; ${ }^{a}$ iszhyao@buaa.edu.cn
}

\begin{abstract}
.
Membrane computing with the advantages of distribution and maximum parallelism is a new branch of natural computing, and discrete Morse theory is a very useful tool in topology. In this paper, combining discrete Morse theory and membrane computing, a novel membrane structure- $\mathrm{P}$ system with chain structure is proposed, which is constructed on the basis of discrete gradient vector path. A specific application example, $\mathrm{P}$ chain system for arithmetic operation, is introduced too. By simulation of it, the superiority, computational efficiency and ability of P system with chain structure are verified.

Keywords: P System; Discrete Morse Theory; Operation Design; Simulation

\section{Introduction}

Membrane computing is a new computational model proposed by Paun in 1998 . It abstracts cell as the computational unit, permitting each computational unit to calculate dependently and the whole system to operate in the way of maximum parallelism, whose computational efficiency has been improved obviously. It has been proved that the computational ability of membrane computing is equivalent with that of Turing machines, so due to its strong parallel computing power it has been the highlight of the recent study [1].

Morse theory as a useful tool in differential topology, particular for computer graphics, was extended by Forman to the discrete aspect [2], which provides an effective tool to describe the topology of discrete object. Concepts in discrete
\end{abstract}


Morse theory, such as simplex, discrete gradient vector path and so on, provide a useful tool to research the topology of discrete structure.

In literature [4], Paun pointed out that the focus of the next stage of membrane computing study was the non-hierarchical arrangement of membranes. Inspired from literature [5], in this paper, a $\mathrm{P}$ system with chain structure is introduced, which combines membrane computing with discrete Morse theory and constructs a $\mathrm{P}$ system based on the discrete gradient vector path, forming a new kind of non-hierarchical membrane structure-P chain system.

\section{P system with chain structure}

\section{Description of structure, object and rule of $\mathbf{P}$ chain system}

For the structure of $\mathrm{P}$ chain system, we know that it is non-hierarchical, specifically it is a chain structure based on discrete gradient vector path. Fig. 1 shows a discrete gradient vector path of a complex and a P chain system based on it. While as for the object, it is similar with the former $\mathrm{P}$ system, which is denoted by multisets. But considering the oriented property of simplex, P chain system has the orientation too, meaning there are two kinds of membranes, positive and negative one, so are objects, including $\alpha$ and $\alpha$ ' which represent objects in positive and negative membrane. Note that $\alpha$ and $\alpha$ ' are antimatter, meaning they can't coexist, when they encounter with each other, they will counteract immediately, similar with positive and negative spikes in spiking neural P system.

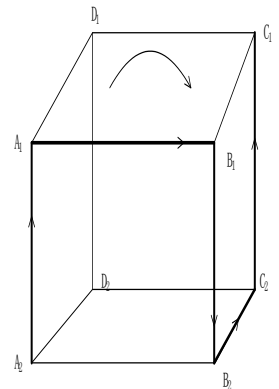

a. Complex $A_{1} B_{1} C_{1} D_{1} \cdot D_{2} A_{2} B_{2} C_{2}$

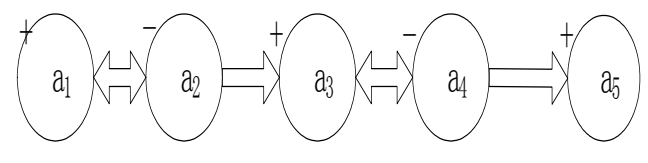

b. P Chain System Based on Discrete Gradient Vector Path $A_{2} A_{1} \rightarrow A_{1} B_{1} \rightarrow B_{1} B_{2} \rightarrow B_{2} C_{2} \rightarrow C_{2} C_{1}$

Fig.1 P System with Chain Structure 
Based on the rules of former $\mathrm{P}$ system, combining the particularities of $\mathrm{P}$ chain system, there are three main rules of $\mathrm{P}$ chain system, rewriting rules, communication rules and forgetting rules.

Rewriting rule is with the form of $u \rightarrow v$, where $u$ is a string representing object multisets from a given set $O$, and $v$ is a string over $O^{*}\{$ here, out, in $\}$, where "here", "out", "in" indicate remaining in the membrane, leaving to its subsequence, and being sent out to its precursor. Rewriting rules are used to control the type and number of objects in membrane.

Communication rules are applied to manage the communication across the membranes, including symport rules, which are formed as $\left(u,\left(\operatorname{tar}_{1}, \operatorname{tar}_{2}\right)\right)$ and involve in objects in one membrane, and antiport rules, which are formed as $(u$, $\left.\left(\operatorname{tar}_{1}, \operatorname{tar}_{2}\right) ; v,\left(\operatorname{tar}_{1}, \operatorname{tar}_{2}\right)\right)$ and control the object communication among membranes. tar $_{1}=\{$ here, out, in $\}$ and $\operatorname{tar}_{2}=\{$ pre, sub $\}$ are target indications and stipulate the direction of object communication, where "here", "out", "in" mean staying in the membrane, going out from the membrane and coming into the membrane, in addition, "pre" and "sub" indicate that move to the precursor and subsequence of the membrane respectively.

Forgetting rule is defined as the form of $u \rightarrow \lambda$, where $u$ is object from multisets, and $\lambda$ is null. Its function is to disappear some certain objects in membrane. A typical example of forgetting rule is when objects $\alpha$ and $\alpha$ ' encounter, they will counteract immediately.

\section{$P$ chain system for arithmetic operation}

The calculating power of P system has been concerned and researched generally, and previous study has proved that NP-hard problem can be resolved by $\mathrm{P}$ system in polynomial time. Literature [5] has proved the possibility of fulfilling arithmetic operation by $\mathrm{P}$ system. Based on this, $\mathrm{P}$ chain system for arithmetic operation is proposed here, compared with the former method, there are some improvements. The specific formalization expression and $\mathrm{P}$ chain system structure are shown in Table 1. And the comparison of time complexity between our method and one in Literature [5] is displayed in Table 2. 
Table 1 P Chain System for Arithmetic Operation

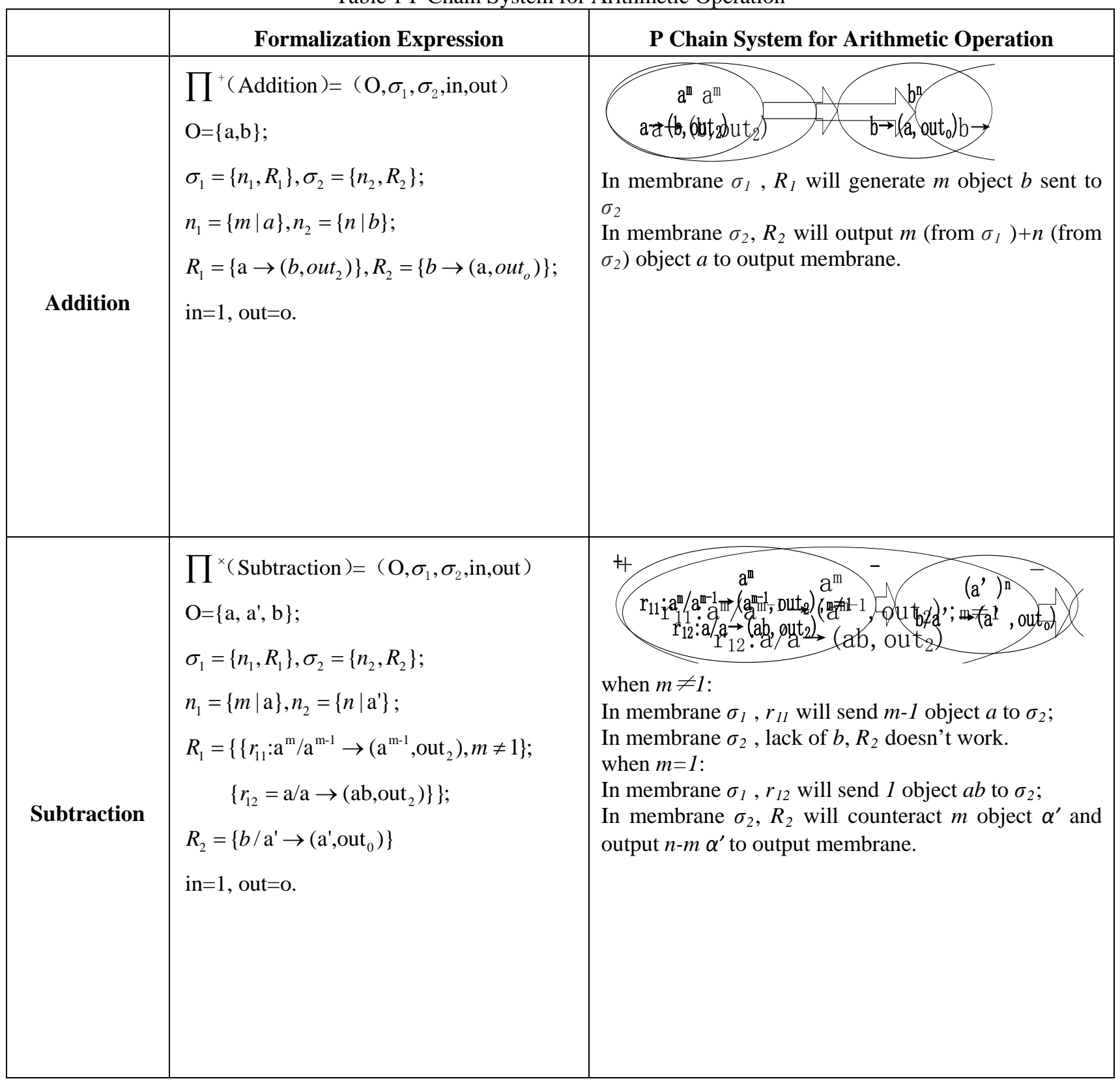




\begin{tabular}{|c|c|c|}
\hline Multiplication & $\begin{array}{l}\prod^{+}(\text {Multiplication })=\left(\mathrm{O}, \sigma_{1}, \sigma_{2}, \text { in,out }\right) \\
\mathrm{O}=\{\mathrm{a}, \mathrm{b}\} \\
\sigma_{1}=\left\{n_{1}, R_{1}\right\}, \sigma_{2}=\left\{n_{2}, R_{2}\right\} \\
n_{1}=\{m \mid a\}, n_{2}=\{0 \mid \phi\} \\
R_{1}=\left\{\mathrm{a} \rightarrow\left(\mathrm{a}, \text { uut }_{2}\right)\right\} \\
R_{2}=\left\{r_{21}: \mathrm{a} \rightarrow \mathrm{b}^{\mathrm{n}} ; r_{22}: \mathrm{b}^{\mathrm{n}} \rightarrow\left(\mathrm{b}^{\mathrm{n}}, \text { out }_{0}\right)\right\} ; \\
\text { in=1, out }=\mathrm{o}\end{array}$ & $\begin{array}{l}\mathrm{a} \rightarrow\left(\mathrm{a}, \text { out }_{2}\right) \\
\text { In membrane } \bar{\sigma}_{1}, R_{1} \text { will send } m \text { object } a \text { to } \sigma_{2} ; \\
\text { In membrane } \sigma_{2}, r_{21} \text { will produce } m b^{n} ; \\
r_{22} \text { will output } m^{*} \text { object } b \text { to output membrane. }\end{array}$ \\
\hline Division & $\begin{array}{l}\prod^{+}(\text {Division })=\left(\mathrm{O}, \sigma_{1}, \sigma_{2}, \text { in }, \text { out }\right) \\
\mathrm{O}=\{\mathrm{a}, \mathrm{b}\} ; \\
\sigma_{1}=\left\{n_{1}, R_{1}\right\}, \sigma_{2}=\left\{n_{2}, R_{2}\right\} ; \\
n_{1}=\{m \mid a\}, n_{2}=\{0 \mid \phi\} ; \\
R_{1}=\left\{\mathrm{a}^{\mathrm{n}} \rightarrow\left(\mathrm{a}^{\mathrm{n}}, \text { out }_{2}\right)\right\}, \\
R_{2}=\left\{r_{21}: a^{n} \rightarrow b ; r_{22}: b \rightarrow\left(\mathrm{b}, \text { out }_{\mathrm{o}}\right)\right\} ; \\
\text { in=1, out }=0 .\end{array}$ & $\begin{array}{l}\text { In membrane } \sigma_{1}, R_{1} \text { will send } k^{*} n \text { object } a \text { to } \sigma_{2} \\
\left(k^{*} n \leq m\right) ; \\
\text { In membrane } \sigma_{2}, r_{21} \text { will produce } k \text { object } b ; \\
r_{22} \text { will output } k \text { object } b \text { to output membrane. }\end{array}$ \\
\hline
\end{tabular}

Table 2 Comparison of time complexity of two methods

\begin{tabular}{ccccc}
\hline $\begin{array}{c}\text { Time } \\
\text { complexity }\end{array}$ & Addition & Subtractio & Multiplicatio & Division \\
\hline Literature[5 & $O(n+m+1)$ & $O(n+m+1)$ & $O(m)$ & $O(n+m+q+1+1$ \\
] & & & & ) \\
This paper & $O(\max \{m, n\}$ & $O(n)$ & $O(m+2)$ & $O(m / n+2)$ \\
& & & & \\
\hline
\end{tabular}




\section{Simulation of arithmetic operation $\mathbf{P}$ chain system}

The simulation of $\mathrm{P}$ system has a vital practical significance, and there has been a series of success $\mathrm{P}$ system simulation software which can be obtained from $\mathrm{P}$ system web page [6]. Based on this, in this paper, we try to simulate $\mathrm{P}$ chain system for arithmetic operation, as a result, it has been proven feasible. We store the object multisets, rules and structure of the $\mathrm{P}$ chain system in the system by the form of nonnegative integer, strings and linear structure respectively. On the other hand, we refer to the chained-rules [7] and principle of weak relationship as the main idea of our Rule Selection Algorithm. We use Microsoft Visual Studio 2008 to fulfill it on Windows 7. Fig.2 is the demonstration of simulation of $\mathrm{P}$ chain system for arithmetic operation.
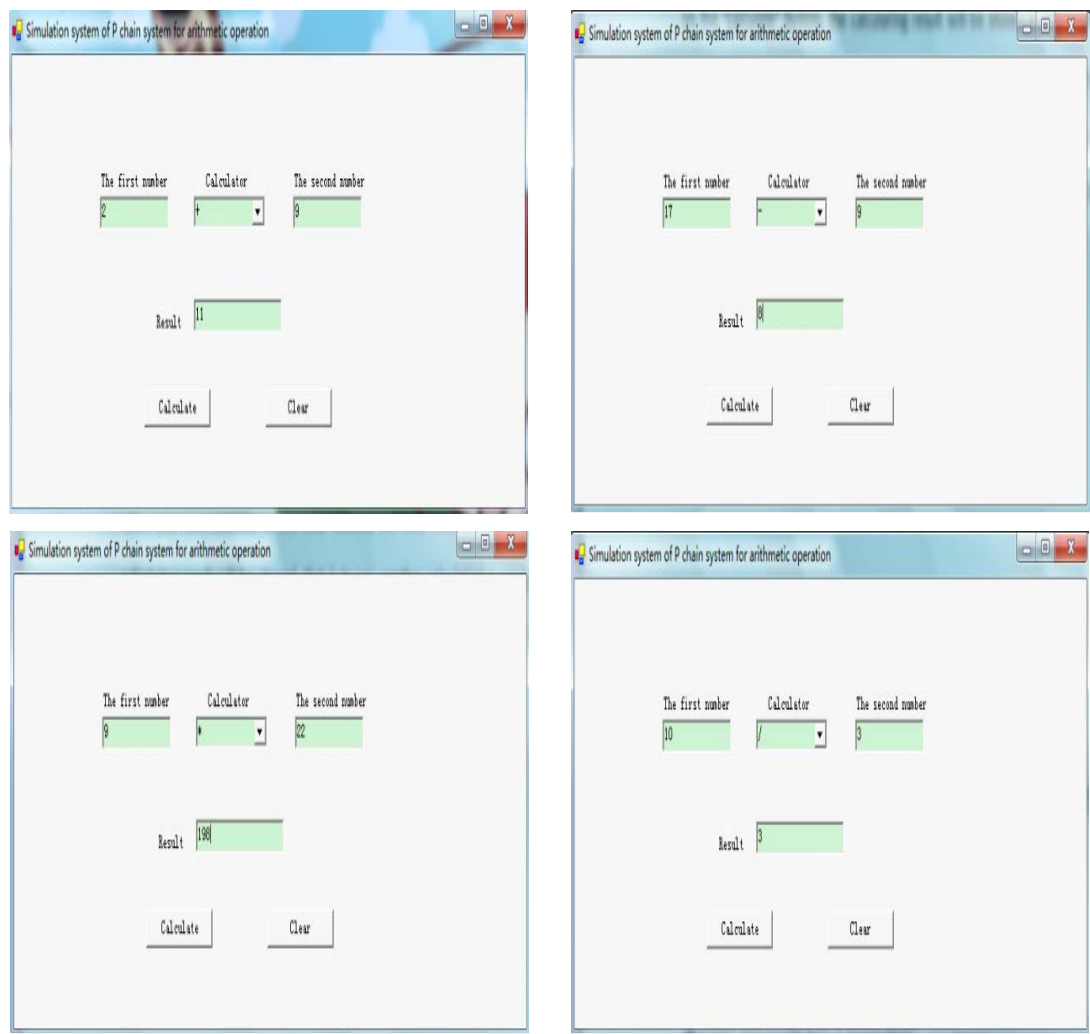

Fig.2 Simulation system of P Chain System for arithmetic Operation 


\section{Summary and prospect}

In this paper, a novel $\mathrm{P}$ system-P system with chain structure, and its specific application example-P chain system for arithmetic operation are introduced. Then its simulation demonstrates the computational feasibility and effectiveness of $\mathrm{P}$ chain system. Because it is a new attempt to construct new type of $\mathrm{P}$ system with non-hierarchical structure, work in this paper is not enough, and there are many aspects need go on further, including more theoretical and practical investigation of P chain system.

\section{Acknowledgements}

This work has been supported by the Natural Science Fundation of China (Project No. 71271012, 71071006, 71332003).

\section{References:}

[1] G. Paun. Membrane Computing. Fundamentals of Computing Theory, 2003, 2751: pp284-295.

[2] R. Forman. Theory for Cell Complexes. Advances in Mathematics, 1998, vol. 134, pp90-145.

[3] G. Paun. An Introduction to and an Overview of Membrane Computing. The Journal of Logic and Algebraic Programming, 2010, vol. 79, no. 6, pp. 291-294.

[4] Xiyu Liu, Alice Xue. Communication P Systems on Simplicial Complexes with Applications in Cluster Analysis. Discrete Dynamics in Nature and Society, 2012, vol. 2012, pp1-17.

[5] Ping Guo, Jing Chen. Arithmetic Operation in Membrane System. 2008 International Conference on BioMedical Engineering and Informatics (BMEI), 2008, vol. 1, pp231-234.

[6] The P systems Web Page: http://ppage.psystems.eu/ visited at August 10, 
2014.

[7] Dragos Sburlan. P Systems with Chained Rules. Twelfth International Conference on Membrane Computing, Lecture Notes in Computer Science, 2012, vol. 7184, pp 359-370. 